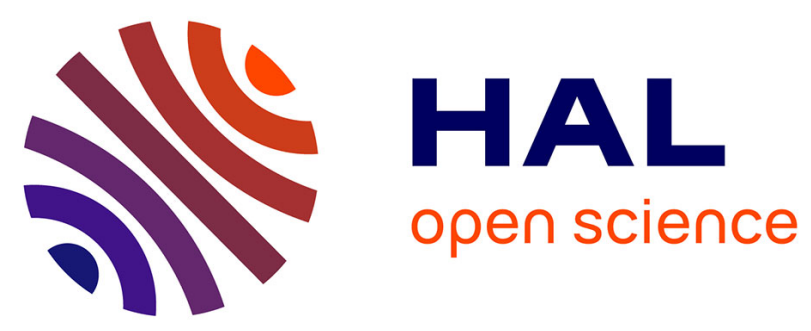

\title{
Mechanical response of human abdominal walls ex vivo: Effect of an incisional hernia and a mesh repair
}

Florence Podwojewski, Mélanie Ottenio, Philippe Beillas, Gaëtan Guerin, Frédéric Turquier, David Mitton

\section{- To cite this version:}

Florence Podwojewski, Mélanie Ottenio, Philippe Beillas, Gaëtan Guerin, Frédéric Turquier, et al.. Mechanical response of human abdominal walls ex vivo: Effect of an incisional hernia and a mesh repair. Journal of the mechanical behavior of biomedical materials, 2014, 38, pp.126-133. 10.1016/j.jmbbm.2014.07.002 . hal-01417372

\section{HAL Id: hal-01417372 \\ https://hal.science/hal-01417372}

Submitted on 15 Dec 2016

HAL is a multi-disciplinary open access archive for the deposit and dissemination of scientific research documents, whether they are published or not. The documents may come from teaching and research institutions in France or abroad, or from public or private research centers.
L'archive ouverte pluridisciplinaire HAL, est destinée au dépôt et à la diffusion de documents scientifiques de niveau recherche, publiés ou non, émanant des établissements d'enseignement et de recherche français ou étrangers, des laboratoires publics ou privés. 
Mechanical response of human abdominal walls ex vivo: Effect of an incisional hernia and a mesh repair

F. Podwojewski ${ }^{1}$, M. Otténio ${ }^{1}$, P. Beillas ${ }^{1}$, G. Guérin ${ }^{2}$, F. Turquier ${ }^{2}$, D. Mitton ${ }^{1, *}$

1 Université de Lyon, F-69622, Lyon; IFSTTAR, LBMC, UMR_T9406 ; Université Lyon 1, France

2 Covidien, Trévoux, France

* Corresponding author: LBMC - Site Ifsttar Lyon-Bron, 25 Av. F. Mitterrand, Case 24, 69675 Bron Cedex, France. Tel.: +33 472142361

E-mail address: david.mitton@ifsttar.fr (D. Mitton). 


\begin{abstract}
The design of meshes for the treatment of incisional hernias could benefit from better knowledge of the mechanical response of the abdominal wall and how this response is affected by the implant. The aim of this study was to characterize the mechanical behaviour of the human abdominal wall. Abdominal walls were tested ex vivo in three states: intact, after creation of a defect simulating an incisional hernia, and after reparation with a mesh implanted intraperitonally. For each state, the abdominal wall was subjected to air pressure loading. Local strain fields were determined using digital image correlation techniques. The strain fields on the internal and external surfaces of the abdominal wall exhibited different patterns. The strain patterns on the internal surface appeared to be related to the underlying anatomy of the abdominal wall. Higher strains were observed along the linea alba than along the perpendicular direction. Under pressure loading, the created incision increased the strain of the abdominal wall compared to the intact state in 5 cases of a total 6 . In addition, the mesh repair decreased the strains of the abdominal wall compared to the incised state in 4 cases of 6. These results suggest that the intraperitoneal mesh restores at least partially the mechanical behaviour of the wall and provides quantification of the effects on the strains in various regions.
\end{abstract}




\section{Introduction}

The treatment of incisional hernias by synthetic mesh implantation has contributed to the reduction of recurrence rates from 25-55\% to 2-25\% (Korenkov et al., 2001; Heniford et al., 2003; Cobb et al., 2003). However, patients may still suffer from pain or limited mobility of the abdomen (McLanahan et al., 1997; Müller et al., 1998). To reduce pain in patients, it has been suggested that an abdominal wall with an implant will demonstrate similar behaviour to the intact abdominal wall (Junge et al., 2001; Hernández et al., 2011; Konerding et al., 2011). Thus, a better knowledge of the biomechanical response of the abdominal wall and how it is affected by the implant could be helpful to further improve the treatment of incisional hernias. However, few studies considered the behaviour of an intact abdominal wall, and even fewer examine the behavior while interacting with an implant. Indeed, many studies only focused on the response of some components of the abdominal wall using an ex vivo sample testing approach. For example, Hwang et al. (2005) and Hernandez et al. (2011) studied the behaviour of the abdominal muscles on animals. Some authors conducted tensile tests on the human linea alba and rectus sheath (Rath et al. (1997), Hollinsky and Sandberg (2007), Förstemann et al. (2011), Martins et al. (2012), Ben Abdelounis et al. (2013)). In addition, the mechanical tests are often far from physiological loading conditions. Junge et al. (2001) only considered uniaxial loading to study the abdominal wall elasticity (with skin and adipose tissue removed). Song et al. (2006), Van Ramshorst et al. (2011) and Szymczak et al. (2012) studied the complete human abdominal wall in vivo, which made the loading conditions more physiological, but limited the analysis to the skin surface of the abdominal wall. No data could be collected on the internal surface of the abdominal wall, which is where incisional hernias occurred and where meshes are typically implanted. Finally, few studies evaluated the influence of a mesh on the mechanical response of the abdominal wall and assessed the differences with the response of a healthy abdominal wall (Müller et al., 1998; Hernández- 
Gascón et al. (2012)). Müller et al. reported the effect of implanted meshes on the abdominal wall mobility measured noninvasively using three-dimensional stereography and compared with a non-operated healthy control group. However, this study is limited to external observations. Hernández-Gascón et al. tested ex vivo abdominal wall tissue specimens from rabbits (one group with meshes, one group as a control). The loading was limited to uniaxial loadings.

Finally, note that no information on an incisional hernia effect on the human abdominal wall behaviour is given in the literature.

A recent study from our group proposed a methodology to assess the behaviour of the abdominal wall under three states: intact, incised, and repaired with a mesh (Podwojewski et al., 2013). However, the methodology was developed and applied on porcine abdominal walls. The objective of the current study is to characterize, for the first time, the mechanical behaviour of the human abdominal wall using this methodology. The internal surface of the abdominal wall (location of the incisional hernias) and the external surface of the abdominal wall (the only visible one in vivo) were studied simultaneously.

\section{Materials and methods}

\subsection{Specimen \& preparation}

Six anterolateral abdominal walls were dissected from post-mortem human subjects (2 female and 4 male subjects, all 66 to 84 years old) obtained from the Department of Anatomy Rockefeller of the University of Lyon (Lyon, France) through the voluntary body donation to science program. The abdominal walls were first dissected along the xiphoid process, the costal margins, the pubic bones, and the iliac crests. The lateral incisions were made between the iliac spines and the lowest point of the rib cage. All layers of the abdominal wall were preserved: skin, adipose tissue, aponeuroses, muscles, and peritoneum. Specimens were 
collected over a period of 14 months and then kept frozen at $-20^{\circ} \mathrm{C}$. They were thawed at room temperature for 16 hours before the test. Just before testing, the external surface of the abdominal wall was shaved when necessary. The height, the width, and the average thickness of each abdominal wall were also measured and are reported in Table 1 . At the beginning of the experiment, the specimens were sprayed with saline solution to prevent drying.

Table 1. Age and gender of the subjects. Dimensions $(\mathrm{cm})$ and average thickness $(\mathrm{mm})$ of the abdominal walls

\begin{tabular}{lllll}
\hline Abdominal wall & $\begin{array}{l}\text { Age (years) / } \\
\text { Gender (M/F) }\end{array}$ & Height $(\mathrm{cm})$ & Width $(\mathrm{cm})$ & Thickness $(\mathrm{mm})$ \\
\hline H1 & $66 / \mathrm{M}$ & 25 & 38 & 25 \\
H2 & $67 / \mathrm{M}$ & 28 & 37 & 17 \\
H3 & 82/F & 25 & 38 & 33 \\
H4 & $77 / \mathrm{M}$ & 31 & 33 & 19 \\
H5 & 84/M & 26 & 42 & 12 \\
H6 & $77 / F$ & 30 & 34 & 20 \\
\hline Mean & 75.5 & 27.5 & 37 & 21 \\
SD & 7.5 & 2.4 & 2.9 & 6 \\
\hline
\end{tabular}

\subsection{Experimental setup}

The experimental setup is based on the protocol described in Podwojewski et al. (2013), using air pressure loading. The main steps and the specificity of this study on human specimens are given below. First, the edges of the abdominal wall were tightened between an aluminium plate and a rubber ring using custom designed clamps. The custom grips illustrated Figure 1 are composed of:

- $\quad$ on one side, an aluminium plate (with an octagonal hole). The border of the hole is covered by a water resistant sandpaper and a plastic tube is inserted in a groove to strengthen the gripping effect. 
- $\quad$ on the other side a rubber ring covers the abdominal wall. The side in contact with the abdominal wall is covered by a water resistant sandpaper.

- $\quad$ clamps to tighten the abdominal wall in between the plate and the ring (Figure 1.b). Octagonal holes in the aluminium plate and in the rubber sheet enabled exposure of both surfaces of the abdominal wall (Figure 1).

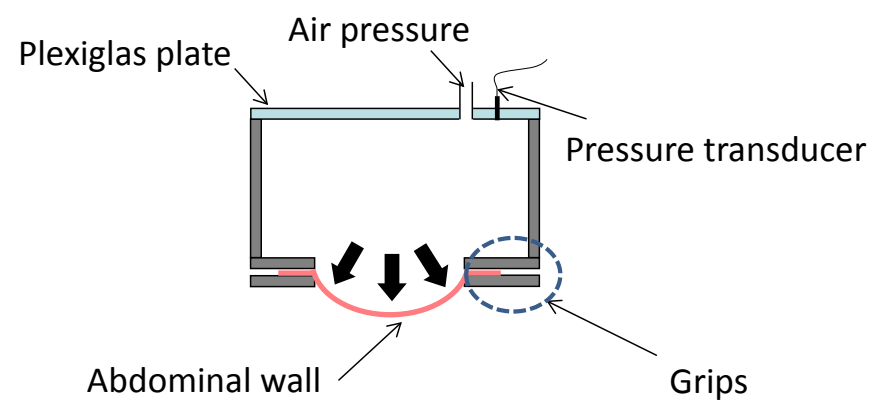

$\mathrm{a}$
(external surface)
(aluminium plate, rubber ring and clamps)
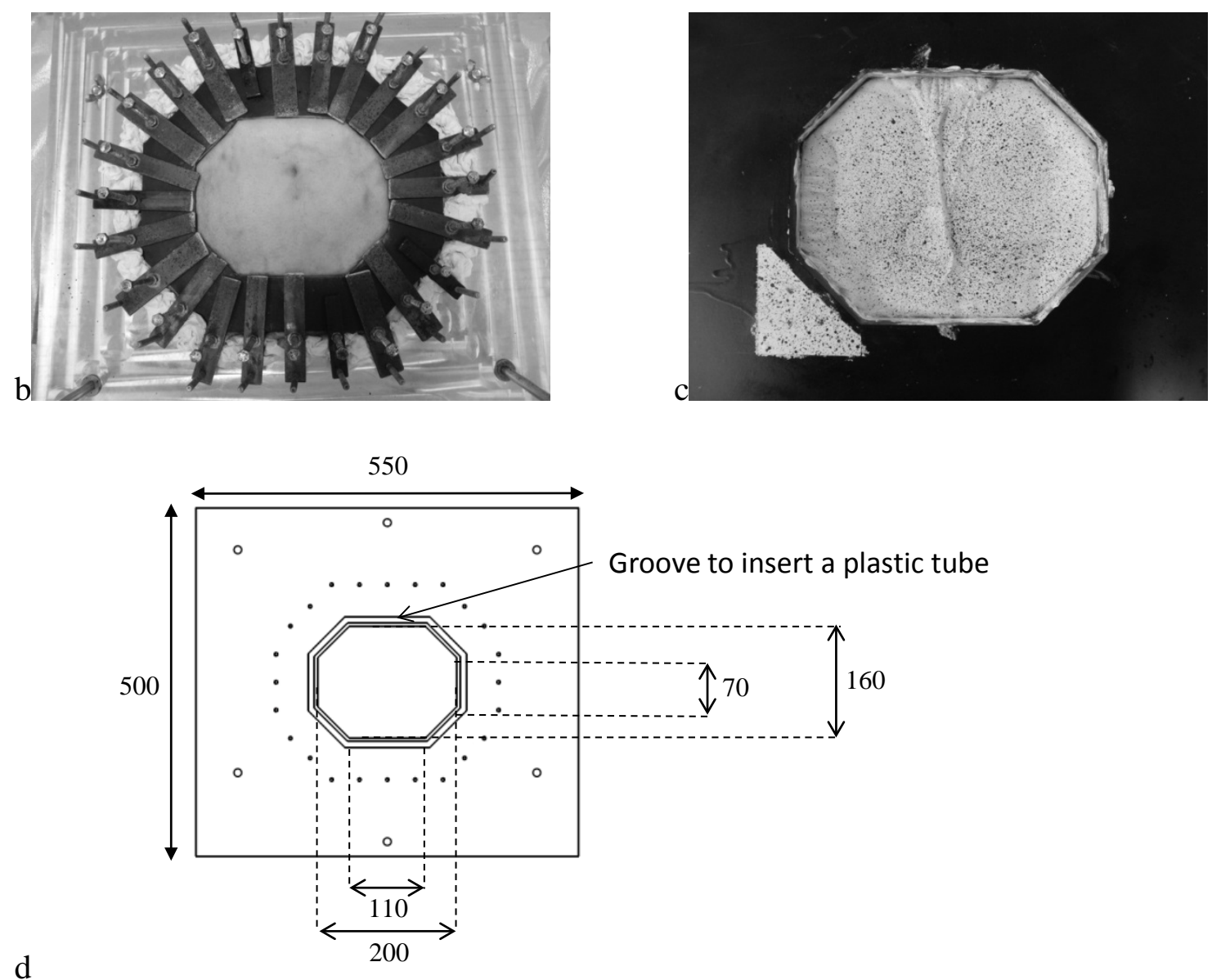

Figure 1. a) Scheme of the custom test apparatus. b) View of the grips exposing the external surface (prior to the application of the speckle) with aluminium plate, rubber ring and clamps 
and (c) the internal surface (with the speckle). d) Schematic drawing of the aluminum plate with the octagon defining the loaded area of the abdominal wall. The groove allows inserting a plastic tube to strengthen the gripping effect between the abdominal wall and this plate. The black dots represent the threaded holes used to tighten the clamps.

As human abdominal walls differed from porcine abdominal walls in size and shape, the shape of the hole was modified to an octagon. The octagonal shape was used to ensure that the largest area can be loaded for all the specimens considered.

Then the abdominal wall on its support plate was positioned on a custom-designed aluminium table. A Plexiglas plate was mounted on the top of the table in order to create a closed cavity above the internal surface of the abdominal wall. Compressed air and a manual valve were used to control the pressure in the cavity. The abdominal wall was then subjected to air pressure loading by applying in the cavity a pressure of $50 \mathrm{mmHg}$ (loading in 9s and unloading in $12.5 \mathrm{~s}$ in average), which is the average measured pressure during the Valsalva manoeuvre (Cobb et al., 2005). The pressure loading cycle was repeated six times to precondition the wall. Only the last cycle was analysed.

The six abdominal walls were successively loaded by pressure for three states: intact, incised, and repaired. For the incised state, a 4cm-long incision was made on the mid-sagittal line of the linea alba. The incision was left open, filled with Vaseline, and covered with a latex film to avoid air infiltrations between the layers of the abdominal wall. For the repaired state, the abdominal wall was repaired with Parietex ${ }^{\mathrm{TM}}$ Composite meshes (Covidien, Trévoux, France), whose dimensions were adjusted to $8 \mathrm{~cm}$ by $12 \mathrm{~cm}$ in order to let an overlap between the defect and the mesh of at least $3 \mathrm{~cm}$. This is in agreement with the recommendation from Cobb et al. (2003) and Binnebösel et al. (2007) to reduce the risk of recurrence. The meshes were fixed on the wall with 16 tacks (AbsorbaTack ${ }^{\mathrm{TM}}$, Covidien, Trévoux, France) that were positioned at one centimetre of the edge of the implant and spaced $2 \mathrm{~cm}$ apart. The meshes 
were also covered with a soft plastic film $(13 \mathrm{~cm}$ by $17 \mathrm{~cm}$, thickness $10 \mu \mathrm{m})$ to avoid air infiltration between the implant and the peritoneum. The plastic film was laid on the abdominal wall and left free to slide. It was maintained only by the applied pressure, and it was assumed that it had no mechanical effect.

\subsection{Measurements}

The pressure applied to the abdominal wall was measured by a pressure sensor (ENTRAN EPX-N02). During the deformation, videos of the internal and external surfaces of the abdominal wall were recorded with four synchronized SA3 PHOTRON black-and-white cameras (Tokyo, Japan). Two cameras equipped with 35mm Zeiss lenses (Oberkochen, Germany) were set on the internal surface, and the two others (equipped with $24-70 \mathrm{~mm}$ Sigma lenses (Tokyo, Japan) in $24 \mathrm{~mm}$ position) were set on the external surface. The resolution of the cameras was 1024 by 1024 pixels, which allocated approximately 4 pixels per $\mathrm{mm}$ in the region of interest. The acquisition frequency was 10 frames per second. Both surfaces of the abdominal wall were covered with white makeup to create a uniform background, and a speckle pattern was applied with black spray paint (Figure 1c). The speckle was used to determine 3D surface displacements and strain fields by digital image correlation using the VIC3D ${ }^{\circledR}$ stereo-correlation software (Correlated Solution, South Carolina, USA).

The Lagrange first principal strain fields were computed for the three states on the whole external surface of the abdominal wall. The strain fields on the internal surface could only be computed for the intact abdominal wall. For the incised and repaired states, the presence of a plastic film on the internal surface prevented image correlation on this surface. Strains along the longitudinal direction (parallel to the linea alba) as well as transverse (perpendicular to that direction) and oblique were also calculated on the external abdominal wall surface. 


\subsection{Statistical analysis}

A statistical analysis was performed on strain results in order to assess the influence of the state (intact, incised, and repaired) of the abdominal wall. A Wilcoxon non-parametric test (Wilcoxon, 1945) for paired samples was used. A value of $\mathrm{p}<0.05$ was selected to indicate statistical significance. Statistical analysis was performed using the Unistat ${ }^{\circledR}$ software (London, England).

\section{Results}

The protocol was successfully applied on 6 specimens (duration: 4 h each).

\subsection{Influence of a defect and mesh repair on the behaviour of the abdominal wall}

For each abdominal wall and each state, profiles corresponding to a section of the external abdominal wall in the transverse plane in the umbilical area were plotted at the initial pressure and at 50mmHg pressure. For example, the profiles of the abdominal wall $\mathrm{H} 1$ are provided in Figure 2.
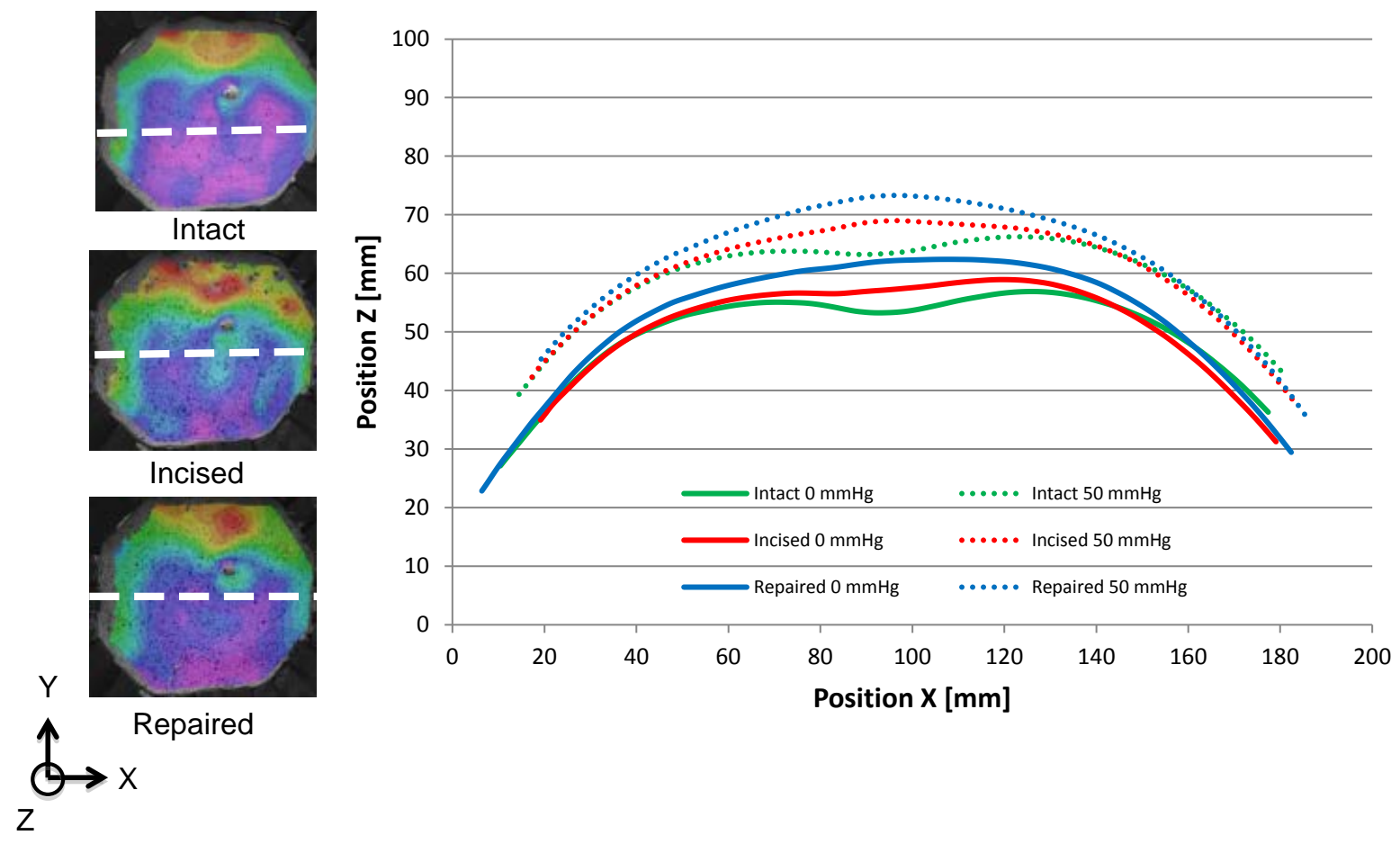
Figure 2. Position profiles of the abdominal wall $\mathrm{H} 1$ in the transversal plane for the three states at a pressure of $0 \mathrm{mmHg}$ and $50 \mathrm{mmHg}$ (external surface).

For five abdominal walls, a concavity at the centre (i.e. near the linea alba) was observed for the intact state. The last wall (H3) had a smoother profile with no concavity (Figure 3) that was associated with a large thickness of adipose tissues and the highest thickness of the walls tested.

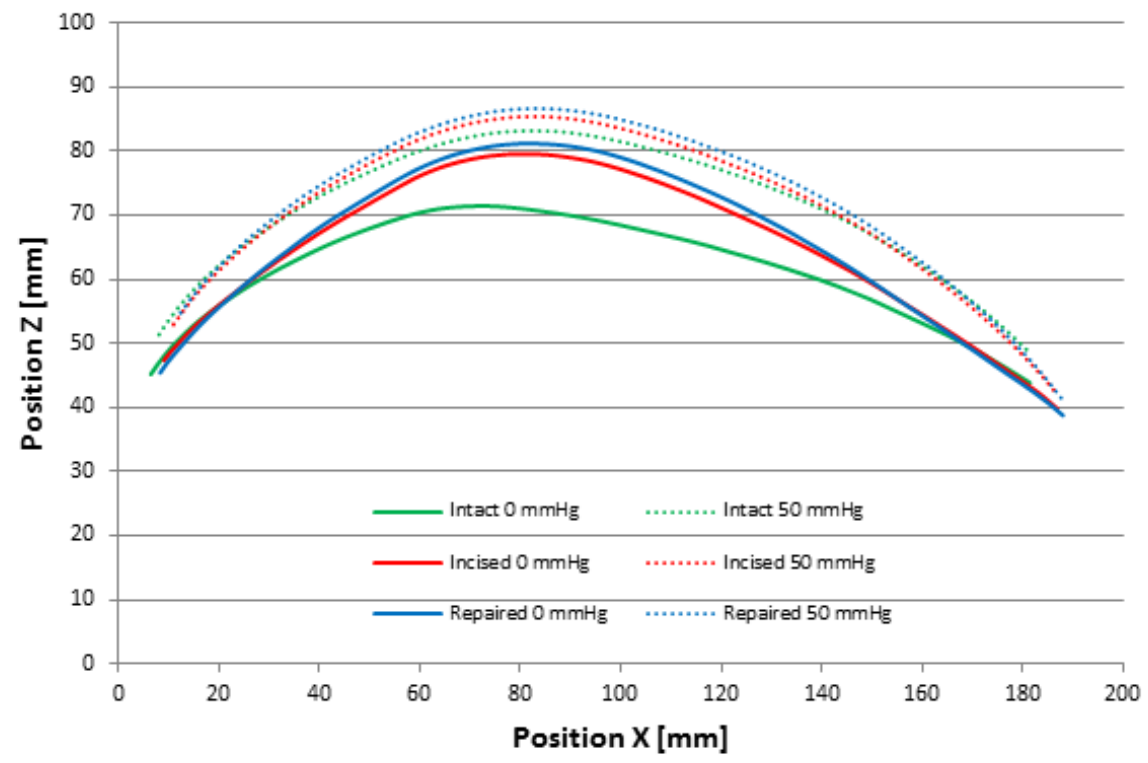

Figure 3. Position profiles of the abdominal wall $\mathrm{H} 3$ in the transversal plane for the three states at a pressure of $0 \mathrm{mmHg}$ and $50 \mathrm{mmHg}$ (external surface)

After incision, the profiles of four abdominal walls (H1, H2, H4, H5) were immediately affected, even without pressure loading. The region in which the concavity was observed in the intact state became flat or even convex (H2, H5). With the increase of the pressure, a larger bump (from 1 to $5 \mathrm{~mm}$ high) comparable to what can be observed in the case of incisional hernia was observed. The repair with a mesh did not seem to reduce the bump. 
Strains calculated at $50 \mathrm{mmHg}$ are reported in Figure 4 for the three states (intact, incised and repaired).
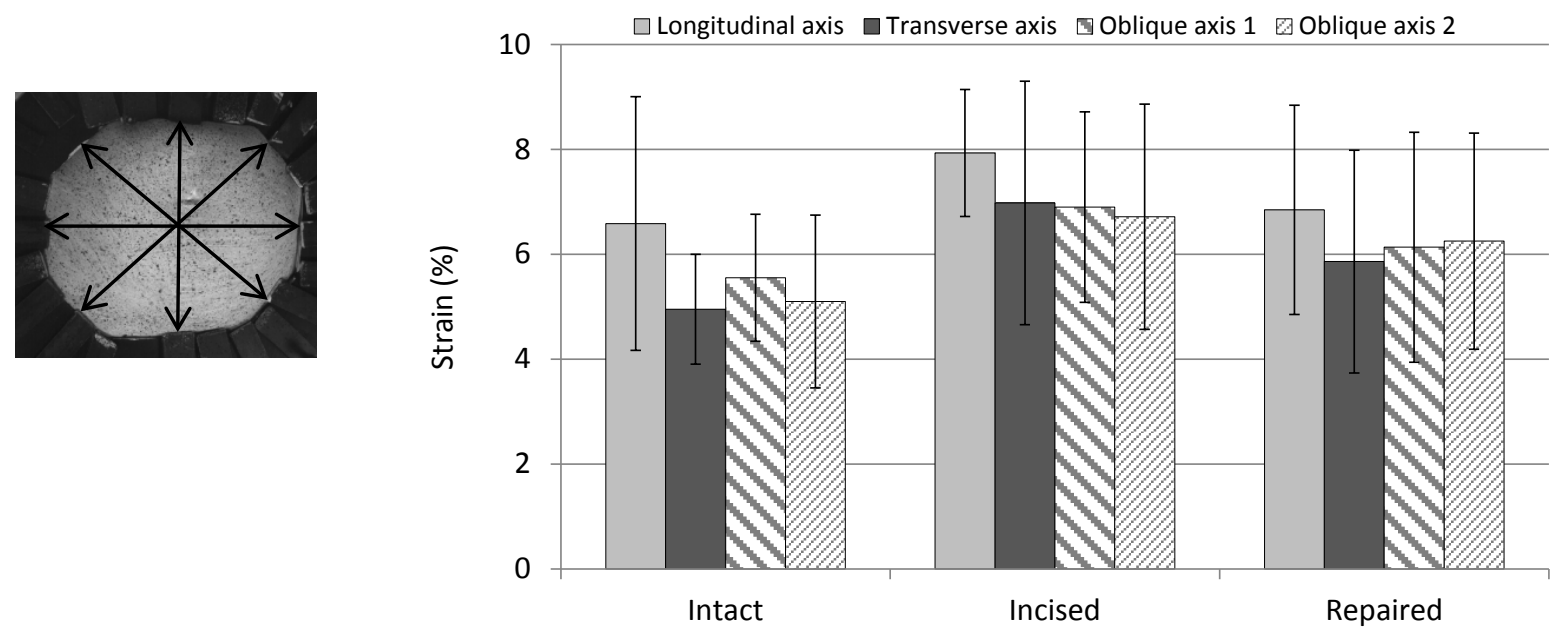

Figure 4. Mean strain of the abdominal wall in longitudinal (L), transverse (T) and oblique $(\mathrm{O} 1, \mathrm{O} 2)$ directions. $\mathrm{N}=6$. Error bars indicate the standard deviation

In the intact state, the average longitudinal and transverse strains at 50mmHg were $6.6 \pm 2.4 \%$ and $5.0 \pm 1.0 \%$, respectively. Corresponding values in the incised state were $7.9 \pm 1.2 \%$ and $7.0 \pm 2.3 \%$. Along the same orientations, the values for the repaired state are $6.8 \pm 2.0 \%$ and $5.9 \pm 2.1 \%$. In all states, the elongation was on average higher in the longitudinal than in the transverse direction. Five abdominal walls (H1, H2, H4, H5, H6) exhibited an increase of the strains after incision of the linea alba while one (H3) did not. Four abdominal walls (H1, H2, H3, H6) exhibited a decrease of the strains after mesh repair; one had the same strain (H5), and one (H4) went from 7.6 to $8.3 \%$. Due to the low number of cases, these tendencies were not significant.

3.2 Relation between internal and external surface strains in the intact abdominal wall 
The maximum locations in the strain field patterns are different in the internal and external surfaces of the abdominal wall. However, strain fields appeared to be symmetrical with respect to the median axis, corresponding anatomically to the linea alba (Figure 5) for the internal surfaces of four abdominal walls.

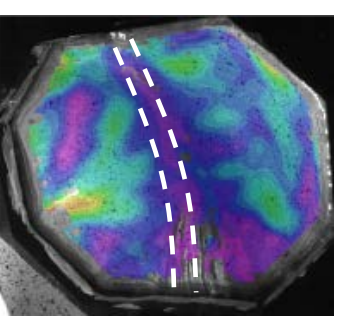

$\mathrm{H} 1$

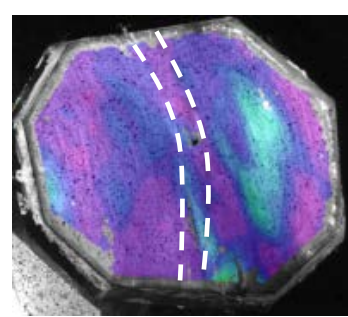

$\mathrm{H} 2$

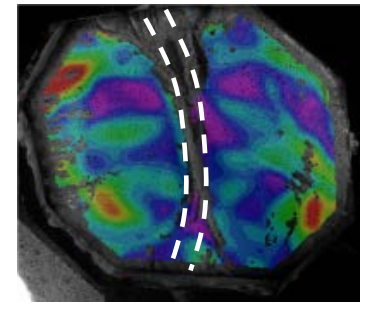

$\mathrm{H} 4$

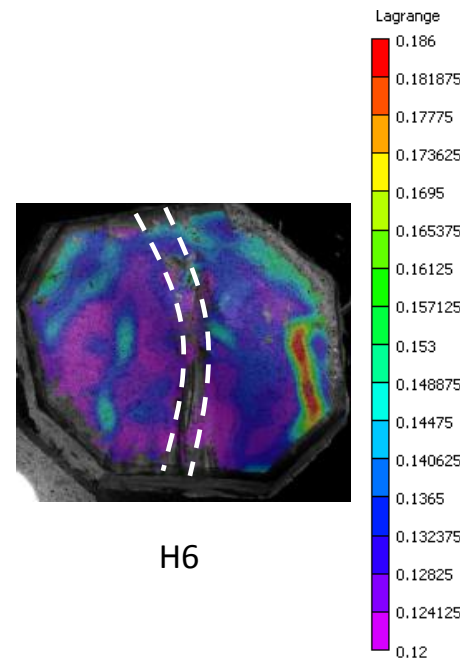

Figure 5. View of the internal surface of the abdominal walls with their strain fields at 50 mmHg - view of the left camera. The dotted lines represent approximately the edges of the linea alba. The dotted lines were placed after anatomical observation The linea alba appears tilted since in digital image correlation, the camera is slightly inclined.

For the two abdominal walls lacking this symmetry, the dissections performed by a visceral surgeon highlighted two issues (Figure 6). In the first case, there was an asymmetry in the positioning of the abdominal wall during tightening; i.e. the linea alba was not centred in the device (Figure 6). In the second case, there was an anatomical abnormality, namely a widening of the linea alba near the centre of the specimen of the abdominal wall (diastasis). 

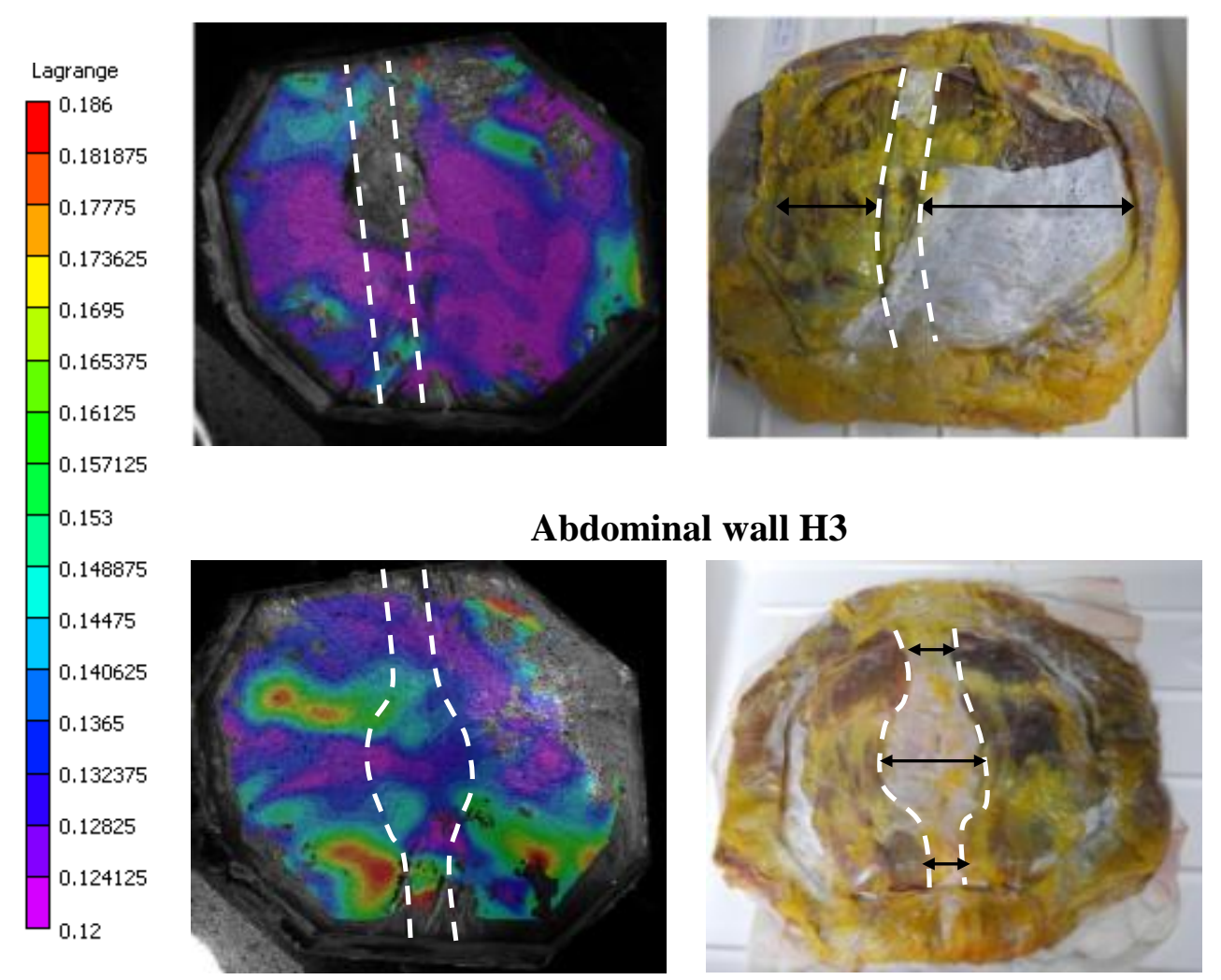

Abdominal wall $\mathbf{H 5}$

Figure 6. Specific cases observed after dissection that may explain the lack of symmetry in the strain fields: the abdominal wall H3 showed an asymmetry related to its position in the experimental device; the abdominal wall H5 had a wider linea alba at the umbilical level.

The mean and standard deviations of the strain values in the longitudinal and transverse directions on the internal and external surfaces at $50 \mathrm{mmHg}$ are summarized in Table 2. 
Table 2. Mean strains (\%) in longitudinal and transverse directions computed on internal and external surfaces of each intact abdominal wall at a pressure of $50 \mathrm{mmHg}$.

\begin{tabular}{|l|ccc|ccc|}
\cline { 2 - 7 } \multicolumn{1}{c|}{ Wall } & $\begin{array}{c}\text { Strain (\%) } \\
\text { Internal } \\
\text { surface }\end{array}$ & $\begin{array}{c}\text { Strain (\%) } \\
\text { External } \\
\text { surface }\end{array}$ & $\begin{array}{c}\text { Ratio } \\
\text { Ext. / Int. }\end{array}$ & $\begin{array}{c}\text { Strain (\%) } \\
\text { Internal } \\
\text { surface }\end{array}$ & $\begin{array}{c}\text { Strain (\%) } \\
\text { External } \\
\text { surface }\end{array}$ & $\begin{array}{c}\text { Ratio } \\
\text { Ext. / Int. }\end{array}$ \\
\hline H1 & 3.9 & 5.8 & 1.5 & 3.1 & 4.4 & 1.4 \\
H2 & 2.5 & 4.6 & 1.8 & 4.1 & 5.1 & 1.2 \\
H3 & 2.5 & 10.5 & 4.1 & 1.8 & 4.9 & 2.7 \\
H4 & 4.7 & 7.4 & 1.6 & 2.2 & 6.1 & 2.8 \\
H5 & 5.3 & 8.2 & 1.6 & 1.6 & 6.1 & 3.9 \\
H6 & 3.0 & 3.1 & 1.0 & 3.0 & 3.1 & 1.0 \\
\hline Mean & 3.7 & 6.6 & 1.9 & 2.6 & 5.0 & 2.2 \\
SD & 1.0 & 2.4 & 1.0 & 0.9 & 1.0 & 1.0 \\
\hline
\end{tabular}

The strains on the external surface were about two times higher than the corresponding strains on the internal surface $(\mathrm{p}<0.05)$ (the ratio (external/internal) is $1.9 \pm 1.0$ and $2.2 \pm 1.0$ for the longitudinal and transverse strains, respectively).

\section{Discussion}

In this study, the experimental protocol developed by Podwojewski et al. (2013) on porcine specimens to characterize the mechanical response of passive abdominal wall in three states (intact, incised, and repaired) was applied to six human abdominal walls. The size and the shape of human abdominal walls after excision differed from the animal specimen and required slight modifications of the protocol. The shape of the hole in the experimental setup exposing the abdominal wall was hence changed to an octagonal shape.

The strains of the human abdominal wall were compared by calculating the mean strain of the abdominal wall along the linea alba, in the transverse, and oblique directions. Higher elongations were obtained along the linea alba than in the transverse direction, which is in agreement with the results obtained ex vivo by Junge et al. (2001) and in vivo by Song et al. (2006) and Szymczak et al. (2012). These results confirm the anisotropic response of the 
abdominal wall and could be used for validation purpose of a finite element model of the abdominal wall.

During pressure loading, the edges of the incision moved away, and a bump was observed on the external surface of the abdominal wall. This protocol was designed to assess the effect of an incision and a mesh repair on the mechanical behaviour of the human abdominal wall. The limited number of specimens that could be tested prevented the study from reaching statistical significance using a Wilcoxon test, as changes between states affected 5 out of the 6 walls, and the presence of the effect on all walls would be required to reach significance. Results should therefore be considered as preliminary tendencies. For the porcine specimen from Podwojewski et al. (2013), the mean strains of the abdominal wall were increased by about 85\% in longitudinal and transverse directions. The mesh repair was associated with mean strains reduction by about $18 \%$ of porcine specimens.

However, the shapes of the region exposed to pressure - triangular for the pig and octagonal for the human - could affect some of these results, and it could be interesting to test the abdominal walls of the two species using the same shape. This could facilitate the comparisons and possibly help determine a relationship between the mechanical responses of the porcine and human abdominal walls. Indeed, such a relationship would be helpful, since animal samples are more accessible than human samples. Moreover, ex vivo mechanical characterization of an abdominal wall repaired with mesh at different post-operative times, like that which was studied by Hernández- Gascón et al. (2012) and Konerding et al. (2012), can only be performed on animal samples. Further work could also include multiple pressure levels, which could facilitate the identification of material parameters in modelling studies, and other states that could be clinically relevant (e.g. add a state with repair by suture only without using a mesh). 
This study also provides results on the strain fields measured simultaneously on the internal and external surfaces of an intact abdominal wall. Analyses showed that the strain patterns differed on the two surfaces. The location of the maximum strain on the internal surface did not correspond to the location of the maxima on the external surface. For the external surface, the strain patterns were homogeneous, while for the internal surface, the strain pattern appeared to be related to the underlying anatomy of the abdominal wall. This suggests that the strain pattern of the internal surface cannot solely be deduced from the strains on the external surface in in vivo studies and that shear or sliding may occur at or between anatomical layers. However, the external strains were found statistically twice higher than the internal ones. In one case, the external strain was four times greater compared to the internal strain. This outlier might be due to the shape specificity of the external part (e.g. umbilicus, initial tension).

For the internal surface, the strain fields appeared to be symmetrical with respect to the longitudinal axis corresponding to the linea alba in 4 out of 6 cases. The lack of symmetry in the other two cases was attributed to positioning or anatomical issues.

Some limitations can be mentioned with regard to the protocol. The effect of the test protocol duration on the response and the effect of the dehydration and rigor mortis were not evaluated. Using a similar setup on the porcine abdominal wall, Podwojewski et al. (2013) found less than $10 \%$ stiffness change between two tests performed at a 3 hours interval. This difference includes duration, and dehydration, among other parameters. While a limited effect was found on the porcine abdominal wall (Podwojewski et al., 2013), it can only be assumed that this result could be transferred to the human samples. The effect of the stiffness of the latex film was not assessed. However the latex film was free to slide on the internal surface. So it can be assumed that its effect is limited. No statistical difference was observed regarding the influence of an incision and a mesh repair. The small number of samples and the presence 
of two atypical cases could explain this result. As for the animal study (Podwojewski at al., 2013), the effect of freezing was not considered. Furthermore, while attention was paid to attempt to minimize the initial stresses in the positioning and tightening, these stresses were not assessed.

In the current study an experimental protocol was applied to observe the effect of an incision and a mesh repair on the biomechanical behaviour of an abdominal wall. This protocol could also detect anomalies on the wall tested (problem of positioning or anatomical anomaly).

A perspective of this study is to perform in vivo experiments in order to characterize the abdominal wall in conditions closer to the physiological conditions. The use of imaging techniques should be considered to observe the internal behaviour of the abdominal wall and not only the external surface.

\section{Acknowledgements}

The authors would like to acknowledge David Voirin from CHU Grenoble for his contribution to this study.

\section{Conflict of interest statement}

This study was partly funded by Covidien Company. G. Guérin and F. Turquier are employees of Covidien France. 


\section{References}

Ben Abdelounis H, Nicolle S, Otténio M, Beillas P, Mitton D. (2013) Effect of two loading rates on the elasticity of the human anterior rectus sheath. Journal of the Mechanical Behavior of Biomedical Materials, 20: 1-5

Binnebösel, M., Rosch, R., Junge,K., Flanagan, T.C., Schwab, R., Schumpelick, V., Klinge, U., 2007. Biomechanical analyses of overlap and mesh dislocation in an incisional hernia model in vitro. Surgery 142 (3), 365-371.

Cobb, W.S., Harris, J.B., Lokey, J.S., McGill, E.S., Klove, K.V., 2003. Incisional herniorrhaphy with intraperitoneal composite mesh: a report of 95 cases. American Surgeon 69 (9), 784-787.

Cobb, W.S., Burns, J.M., Kercher, K.W., Matthews, B.D., James Horton, H., Todd Heniford, B., 2005. Normal intraabdominal pressure in healthy adults. Journal of Surgical Research 129 (2), 231-235.

Förstemann, T., Trzewik, J., Holste, J., Batke, B., Konerding, M.A., Wolloscheck, T., Hartung, C., 2011. Forces and deformations of the abdominal wall - a mechanical and geometrical approach to the linea alba. Journal of Biomechanics 44 (4), 600-606.

Heniford, B.T., Park, A., Ramshaw, B.J., Voeller, G., 2003. Laparoscopic repair of ventral hernias: nine years' experience with 850 consecutive hernias. Annals of surgery 238(3), 391-400.

Hernández, B., Peña, E., Pascual, G., Rodríguez, M., Calvo, B., Doblaré, M., Bellón, J.M., 2011. Mechanical and histological characterization of the abdominal muscle. A previous step to modelling hernia surgery. Journal of the Mechanical Behavior of Biomedical Materials 4 (3), 392-404.

Hernández- Gascón, B., Peña, E., Pascual, G., Rodríguez, M., Bellón, J.M., Calvo, B., 2012. Long-term anisotropic mechanical response of surgical meshes used to repair 
adbdominal wall defects. Journal of the Mechanical Behavior of Biomedical Materials 5 (1), 257-271.

Hollinsky, C., Sandberg, S., 2007. Measurement of the tensile strength of the ventral abdominal wall in comparison with scar tissue. Clinical Biomechanics 22 (1), 88-92.

Hwang, W., Carvalho, J.C., Tarlovsky, I., Boriek, A.M., 2005. Passive mechanics of canine internal abdominal muscles. Journal of Applied Physiology 98, 1829-1835.

Junge „K., Klinge, U., Prescher, A., Giboni, P., Niewiera, M., Schumpelick, V., 2001. Elasticity of the anterior abdominal wall and impact for reparation of incisional hernias using mesh implants. Hernia 5 (3), 113-118.

Konerding, M.A., Bohn, M., Wolloscheck, T., Batke, B., Holste, J.L., Wohlert, S., Trzewik, J., Förstemann, T., Hartung, C., 2011. Maximum forces acting on the abdominal wall: experimental validation of a theoretical modeling in a human cadaver study. Medical Engineering and Physics 33 (6), 789-792.

Konerding, M.A., Chantereau, P., Delventhal, V., Holste, J.L., Ackermann, M., 2012. Biomechanical and histological evaluation of abdominal wall compliance with intraperitoneal onlay mesh implants in rabbits: a comparison of six different state-of-the-art meshes. Medical Engineering and Physics 34 (7), 806-816.

Korenkov, M., Paul, A., Sauerland, S., Neugebauer, E., Arndt, M., Chevrel, JP, Corcione, F., Fingerhut, A., Flament, JB., Kux, M. et al., 2001. Classification and surgical treatment of incisional hernia. Langenbeck’s Archives of Surgery 386(1), 65-73.

Martins, P., Peña, E., Jorge, RM., Santos, A., Santos, L., Mascarenhas, T., Calvo. B., 2012. Mechanical characterization and constitutive modelling of the damage process in rectus sheath. Journal of the Mechanical Behavior of Biomedical Materials 8, 111-122.

McLanahan, D., King, L.T., Weems, C., Novotney, M., Gibson, K., 1997. Retrorectus prosthetic mesh repair of midline abdominal hernia. American Journal of Surgery 173 (5), 
445-449.

Müller, M., Klinge, U., Conze, J., Schumpelick, V., 1998. Abdominal wall compliance after Marlex mesh implantation for incisional hernia repair. Hernia 2,113-117.

Podwojewski, F., Otténio, M., Beillas, P., Guérin, G., Turquier, F., Mitton, D., 2013. Mechanical response of animal abdominal walls in vitro: Evaluation of the influence of a hernia defect and a repair with a mesh implanted intraperitoneally. Journal of Biomechanics, 46 (3), 561-566.

Rath, A.M., Zhang, J., Chevrel, J.P., 1997. The sheath of the rectus abdominis muscle: an anatomical and biomechanical study. Hernia 1,139-142.

Song, C., Alijani, A., Frank, T., Hanna, G., Cuschieri, A., 2006. Elasticity of the living abdominal wall in laparoscopic surgery. Journal of Biomechanics 39 (3), 587-591.

Szymczak, C., Lubowiecka I., Tomaszewska, A., Smietański, M., 2012. Investigation of abdomen surface deformation due to life excitation: Implications for implant selection and orientation in laparoscopic ventral hernia repair. Clinical Biomechanics 27 (2), 105-110.

Van Ramshorst, G.H., Salih, M., Hop, W.C., van Waes, O.J., Kleinrensink, G.J., Goossens, R.H., Lange, J.F., 2011. Non invasive assessment of intra-abdominal pressure by measurement of abdominal wall tension. Journal of Surgical Research, 171 (1), 240-244. 